\title{
COMPACT AND QUASINORMAL COMPOSITION OPERATORS
}

\section{RQA J KISHOR SINGH}

ABSTRACT. Let $C_{\phi}$ be a composition operator on $L^{2}(\lambda)$, where $\lambda$ is a $\sigma$-finite measure on a set $X$. If $X$ is nonatomic, then Ridge proved that no one-to-one composition operator $C_{\phi}$ with dense range is compact. This result is generalized in the paper by removing one-to-one and dense range conditions. The quasinormal composition operators are also characterized in terms of commutativity with the multiplication operator induced by the Radon-Nikodym derivative of the measure $\lambda \phi^{-1}$ with respect to $\lambda$.

1. Introduction. Some results concerning compact and quasinormal composition operators on $L^{2}(\lambda)$ are reported in this paper, where $\lambda$ is a $\sigma$-finite measure on some set $X$. Ridge [1] proved that if $X$ is nonatomic, then no one-to-one composition operator with dense range is compact. We have generalized this result in $\$ 2$ by removing one-to-one and dense range conditions. In $\$ 3$, we have proved a theorem on quasinormal composition operators.

A composition operator $C_{\phi}$ on $L^{2}(\lambda)$ induced by a measurable transformation of $\phi$ on $X$ into itself is defined by $C_{\phi} f \approx f \circ \phi$.

\section{Compact operators.}

Theorem 1. Let $X$ be a nonatomic $\sigma$-finite measure space. Then no composition operator on $L^{2}(\lambda)$ is compact.

First we shall prove the following lemma.

Lemma. Let $X$ be a $\sigma$-finite, nonatomic measure space. Let $E$ be a nonnull measurable subset of $X$. Let $\left\{E_{n}\right\}$ be a sequence of nonnull measurable sets such that $E_{n+1} \subset E_{n} \subset E$ for all $n$, and $\lambda\left(E_{n}\right)<1 / n$. Let $g \in$ $L^{2}(E)$. Then

$$
\left(\lambda\left(E_{n}\right)\right)^{-1 / 2} \int_{E_{n}} g d \lambda \rightarrow 0 \text { as } n \rightarrow \infty
$$

Received by the editors June 5, $197: 3$.

AMS (MOS) subject classifications (1970). Primary 47B05; Secondary $47 \mathrm{~B} 05$.

Key words and phrases. Composition operators, compact operators, quasinormal composition operators, atomic measures, nonatomic measures. 
Proof. Suppose there exists a $g \in L^{2}(E)$ such that $\left(\lambda\left(E_{n}\right)\right)^{-1 / 2} \int_{E_{n}} g d \lambda$ does not tend to 0 as $n$ tends to $\infty$. We can assume that $\|g\|=1$ and $g$ is real valued. Then there exists an $\epsilon>0$ such that

$$
\limsup _{n \rightarrow \infty}\left|\left(\lambda\left(E_{n}\right)\right)^{-1 / 2} \int_{E_{n}} g d \lambda\right|=2 \epsilon>0 .
$$

Let $e_{n}$ denote the function $\left(\lambda\left(E_{n}\right)\right)^{-1 / 2} X_{E_{n}}$. Let $\mathcal{F}=\{n: n \in N$ and $\left.\left|\left\langle g, e_{n}\right\rangle\right|>\epsilon\right\}$. 'Let $F \subset N$. Then by Bessel's inequality we have

$$
\sum_{n \in F}\left|\left\langle g, e_{n}\right\rangle\right|^{2} \leq 1+\sum_{m, n \in F ; m \neq n}\left\langle g, e_{n}\right\rangle\left\langle g, e_{m}\right\rangle\left\langle e_{n}, e_{m}\right\rangle \cdot
$$

Now choose a positive integer $K$ such that $\epsilon^{2}>1: / K$. Select a subset $F$ of $\mathcal{F}$ such that cardinality of $F$ is $2 K$ and $\left\langle e_{n}, e_{m}\right\rangle<(1 / 2)(1 / 2 K(2 K-1))$ for for all $m, n \in F, m \neq n$. From this it follows that $\Sigma_{n \in F}\left|\left\langle g, e_{n}\right\rangle\right|^{2} \geq 2$ and $\Sigma_{n, m \in F ; n \neq m}\left\langle e_{n}, e_{m}\right\rangle \leq 1 / 2$; hence from $(*)$ we obtain $2 \leq 1+1 / 2=3 / 2$, which is a contradiction. Thus proof of the Lemma is complete.

Proof of the theorem. Let $C_{\phi}$ be a composition operator on $L^{2}(\lambda)$. Let $f_{0}$ denote the Radon-Nikodym derivative of the measure $\lambda \phi^{-1}$ with respect to $\lambda$. Since $\lambda$ is nonatomic, we can choose a nonnull subset $E$ such that $f_{0}$ is bounded away from zero on $E$. Also, we can choose a sequence $\left\{E_{n}\right\}$ of $E$ such that $E_{n+1} \subset E_{n}$ and $0<\lambda\left(E_{n}\right)<1 / n$ for all $n$. Consider the sequence $\left\{e_{n}\right\}$ of functions defined by $e_{n}=\left(\lambda\left(E_{n}\right)\right)^{-1 / 2} X_{E_{n}}$. By the previous lemma $e_{n} \rightarrow 0$ weakly. It can be shown that $\left\{C_{\phi} e_{n}\right\}^{n}$ does not tend to 0 strongly. Hence $C_{\phi}$ is not compact. This completes the proof of the theorem.

Corollary 1. Let $\lambda$ be a regular continuous Borel measure on $R$. Then no composition operator on $L^{2}(\lambda)$ is compact.

The following theorem also generalizes a result of Ridge [1].

Theorem 2. Let $X$ be any $\sigma$-finite measure space, and let $C_{\phi}$ be a bounded operator on $L^{2}(\lambda)$. Then $C_{\phi}$ is compact implies that $X$ is an atomic measure space.

Proof. We can write $X=X_{1} \cup X_{2}$ where $X_{1}$ does not contain any atoms, $X_{2}$ is a countable union of atoms and $X_{1}$ and $X_{2}$ are disjoint [3]. Let $\lambda_{2}$ be the restriction of $\lambda$ to $X_{2}$, and let $\lambda_{1}=\lambda-\lambda_{2}$. Since $\lambda \phi^{-1}$ is absolutely continuous with respect to $\lambda$, we can conclude that $X_{2} \subset \phi^{-1}\left(X_{2}\right)$ a.e. If we write $L^{2}(\lambda)=L^{2}\left(\lambda_{1}\right) \oplus L^{2}\left(\lambda_{2}\right)$, then it follows from the above inclusion 
that $C_{\phi} L^{2}\left(\lambda_{1}\right) \subset L^{2}\left(\lambda_{1}\right)$. Since $C_{\phi}$ is compact, from the previous theorem it follows that $\lambda_{1}=0$. Hence $\lambda=\lambda_{2}$. Hence $X$ is an atomic measure space (since $\lambda_{2}$ is an atomic measure). The proof is complete.

3. Quasinormal composition operators.

Theorem 3. A composition operator $C_{\phi}$ on $L^{2}(\lambda)$ is quasinormal if and only if it commutes with $M_{f_{0}}$, where $M_{f_{0}}$ is the multiplication operator induced by $f_{0}\left(x d \lambda \phi^{-1 / d \lambda)}\right.$. $L^{2}(\lambda)$

Proof. First we will prove that $C_{\phi}^{*} C_{\phi}=M_{f_{0}}$. For any $f$ and $g$ in

$$
\left\langle C_{\phi}^{*} C_{\phi} f, g\right\rangle=\left\langle C_{\phi} f, C_{\phi} g\right\rangle=\int f \cdot \bar{g} d \lambda \phi^{-1}=\int f_{0} f \cdot \bar{g} d \lambda=\left\langle M_{f_{0}} f, g\right\rangle .
$$

Hence $C_{\phi}^{*} C_{\phi}=M_{f_{0}}$.

Now suppose $C_{\phi}$ is quasinormal. Then $C_{\phi}^{*} C_{\phi} C_{\phi}=C_{\phi} C_{\phi}^{*} C_{\phi}$, from which we get $M_{f_{0}} C_{\phi}=C_{\phi} M_{f_{0}}$. Conversely, if $C_{\phi} M_{f_{0}}=M_{f_{0}} C_{\phi}$, then, since $M_{f_{0}}=C_{\phi}^{*} C_{\phi}$, we get $C_{\phi} C_{\phi}^{*} C_{\phi}=C_{\phi}^{*} C_{\phi} C_{\phi}$, which shows that $C_{\phi}$ is quasinormal.

\section{REFERENCES}

1. W. C. Ridge, Composition operators, Thesis, Indiana University, 1969.

2. R. K. Singh, Composition operators (to appear).

3. A. C. Zaanen, Integration, Completely revised edition of An introduction to the theory of integration, North-Holland, Am sterdam, Interscience, New York, 196?. MR $36 \# 5286$.

DE PARTMENT OF MATHEMATICS, UNIVERSITY OF NEW HAMPSHIRE, DURHAM, NEW HAMPSHIRE 03824

DE PARTMENT OF MATHEMATICS, UNIVERSITY OF JAMMU, JAMMU, INDIA 